\title{
F(R,T) GRAVITEDE MAKASLAMASIZ MÜKEMMEL AKIŞKAN İDDİASINA İKİNCİ BİR KARŞIT ÖRNEK
}

\author{
(A SECOND COUNTER-EXAMPLE FOR SHEAR-FREE PERFECT \\ FLUID CONJECTURE IN F(R,T) GRAVITY)
}

\section{Değer SOFUOĞLU1}

ÖZ

$\mathrm{Bu}$ çalışmada, değiştirilmiş gravitasyon teorilerinde makaslamasız mükemmel akışkan iddiasına ikinci bir karşıt-örnek bulmak ve benzer çözümlerin $f(R, T)$ gravitede tüm Bianchi tip metrikler için geçerli olup olmadığını incelemek amacıyla, $f(R, T)$ gravitasyon teorisi çerçevesinde, uzayca homojen, eşyönsüz, makaslaması, dönen ve genişleyen Bianchi tip-II ve $\mathrm{V}$ modelleri göz önüne alınmıştır. Bianchi tip-II modeli için kesin bir çözüm elde edilmiş, $f(R, T)$ fonksiyonunun fonksiyonel formu inşa edilmiş ve böyle bir evrenin madde-enerji içeriğinin katı akışkan olduğu gösterilmiştir. Makaslamasız bir mükemmel akışkanla dolu Bianchi tip-V modelinin ise, aynı anda hem dönme ve hem de genişlemeye sahip olamayacağ gösterilmiştir.

Anahtar Kelimeler: Dönme, Genişleme, Makaslamasız, Mükemmel Akışkan, Bianci tip-II Model, Bianchi tip-V Model

\begin{abstract}
In this study, to find the second counter-example for shear-free perfect fluid conjecture in modified gravitation theories and to investigate whether similar solutions are valid for all Bianchi type metrics in $f(R, T)$ gravity; spatially homogeneous and anisotropic, shear-free, rotating and expanding Bianchi type-II and $V$ models have been considered in context of $f(R, T)$ theory of gravity. An exact solution has been obtained, functional form of $f(R, T)$ function has been reconstructed for Bianchi type-II model and it has been shown that the matter-energy content of such a universe is stiff fluid. It has also been shown that Bianchi type-V model filled with a shear-free perfect fluid cannot rotate and expand simultaneously.
\end{abstract}

Keywords: Rotation, Expansion, Shear-free, Perfect fluid, Bianchi type-II Model, Bianchi Type-V Model

\footnotetext{
${ }^{1}$ İstanbul Üniversitesi, Fen Fakültesi, Fizik Bölümü, İSTANBUL, degers@istanbul.edu.tr (sorumlu yazar)
} 


\section{GİRİŞ}

Genel Rölativite Teorisi'nin (GRT) alan denklemlerine dayalı Rölativist Kozmoloji çerçevesinde, makaslamasız mükemmel akışkan iddiası; eğer makaslama sıfır ise, bu takdirde $\mu+p \neq 0$ koşuluna uyan bir mükemmel akışkanın dönmesinin veya genişlemesinin veyahut da her ikisinin sıfır olacağını ifade eder. Başka bir deyişle bu, makaslama olmadığında, rölativist kozmoloji'de hem dönen hem de genişleyen bir evren modeli çözümünün olmayacağı anlamına gelir. Literatürde makaslamasız bir mükemmel akışkanın, birtakım ek varsayımlar sağlandığında, ya dönmesinin ya da genişlemesinin sıfır olduğunu bildiren çok sayıda çalışma bulunmaktadır. Bu yönde ilk sonuç, Gödel tarafından, ispatsız olarak, uzayca homojen modellerden olan Bianchi-tip IX kozmolojik modeli çerçevesinde toz $(p=0)$ hali için verilmiştir [1]. Bu sonuç daha sonra Ellis tarafından yine toz için ama herhangi bir uzayzaman simetrisine sahip olması gerekmeyen tüm modellere genelleştirilmiştir [2]. Bir başka doğrultuda genelleştirme de tüm uzayca homojen uzay-zamanları göz önüne almak ama bu sefer, çok özel hal denklemleri varsaymak yoluyla olmuştur. Bu kapsamda Schücking $p=0$ modellerini [3], Banerji de $p=(\gamma-1) \mu$ lineer barotropik hal denkleminde $1<\gamma \neq \frac{10}{9}$ halini göz önüne almışlardır [4]. Treciokas ve Ellis, hal denklemi $p=\frac{1}{3} \mu$ olan makaslamasız radyasyon modeli için, ya dönmenin ya da genişlemenin sıfır olması gerektiğini ispatlamışlardır [5]. King ve Ellis $\mu$ ve $p$ değişkenlerine yalnızca $\mu+p>0$ kısıtlaması getirilmiş olsa bile sonuçların değiş̧mediğini göstermişlerdir [6]. Makaslamasız durumda ya dönmenin ya da genişlemenin sıfır olduğu başka özel varsayımlar için de gösterilmiştir. White ve Collins, ivmenin dönmeye paralel olması [7] ve Collins de Weyl tansörünün magnetik kısmının sıfır olması durumlarında aynı sonucu bulmuşlardır [8]. Carminati, Petrov tip N ve Petrov tip III durumları için iddianın doğru olduğunu göstermiştir [9-11]. Senovilla ve diğ., 1+3-kovaryant formülasyonu kullanarak, söz konusu iddiayı ivmenin sıfır olduğu ve de ivmenin dönmeye paralel olduğu iki durum için ispatlamaya çalışmışlardır [12]. Bu alandaki çalışmaları başkaları da izlemiştir [13-18]. Konuyla ilgili son gelişmeler ise, yakın zamanda, lineer barotropik hal denklemi $(\gamma \operatorname{nin}-1 / 5,-1 / 6,-1 / 11,-1 / 21,1 / 15,1 / 4$ dışındaki değerleri) için kozmolojik sabitin sıfır olduğu ve daha sonra kozmolojik sabitin sıfırdan farklı olduğu lineer barotropik hal denklemi alınarak yapılan ispatlardır [19,20]. Bununla birlikte, şuna işaret etmekte yarar vardır: Rölativist kozmoloji çerçevesinde, makaslamasız mükemmel akışkan iddiasının koşullarını sağlayan kesin çözümlerin bu iddiayı doğruladıkları bilinmekle birlikte, bu çok özel durumların dışında iddianın tam bir ispatı henüz yapılamamış, dahası bu iddianın yanlış olduğunu gösteren herhangi bir çözüme de şimdiye kadar rastlanamamıştır.

Öte yandan, Newton'un gravitasyon teorisinde ve $f(R)$ gravitede bu iddianın karşılığ bulunmamaktadır; yani, bu teorilerde hem dönen hem de genişleyen makaslamasız mükemmel akışkan çözümleri bulunmaktadır [12,21-23]. Böyle bir durumun varlığı, makaslamasız akışkan iddiasının GRT'nin kendi yapısından kaynaklanıyor olabileceğini ve dolayısıyla gravitasyonel olayları tasvir için GRT'ye alternatif olarak ileri sürülen diğer teoriler çerçevesinde de mümkün karşıt-örnekler bulunabileceği beklentisini telkin etmektedir. Gerçekten de, yakın zamanda bu beklentiyi destekleyen bir çalışma [24], GRT'ye alternatif teorilerden biri olan ve evrenin ileri zamanındaki kozmik ivmesi ve geçmiş zamanındaki 
enflasyonu gibi bir takım kozmolojik meseleleri çözme konusunda son beş yıldan bu yana çok ilgi çekmiş olan $f(R, T)$ gravite teorisi [25] çerçevesinde yapılmıştır. Söz konusu çalışmada, aynı anda hem genişleyen hem de dönen makaslamasız bir mükemmel akışkanla dolu uzaysal olarak homojen ve eşyönsüz Bianchi-tip IX evreninin, $f(R, T)$ gravitenin alan denklemlerinin bir çözümü olduğu gösterilmiş olup bu çözüm, $f(R, T)$ gravite teorisi çerçevesinde makaslamasız mükemmel akışkan iddiasının geçerli olmadığının gösterildiği ilk karşıt-örnek olma özelliğini taşımaktadır.

Biz bu çalışmada, $f(R)$ ve $f(R, T)$ gravitasyon teorilerinin her ikisinde de makaslamasız akışkan iddiasını yanlışladığı bilinen tek karşıt-örnek olan Bianchi-tip IX evren modeli $[23,24], \mu+p \neq 0$ koşuluna uyan bir makaslamasız mükemmel akışkan kaynağı için aynı anda hem dönme ve hem de genişleme gösteren tek model midir sorusundan yola çıkarak, $f(R, T)$ gravite teorisi çerçevesinde makaslamasız mükemmel akışkan iddiasına ikinci bir karşıt-örnek aramak ve de benzer çözümün tüm Bianchi-tip metrikler için geçerli olup olmadığını araştırmak amacıyla, [23]'te kullanılan metodu takip ederek, uzayca homojen ve eşyönsüz metrikler sınıfından olan dönen Bianchi tip-II ve dönen Bianchi tip- $\mathrm{V}$ modellerini göz önüne alıyoruz.

Çalışma planımız şu şekilde olacaktır: 2. Bölüm'de, $f(R, T)$ gravitenin kısa bir sunumu yapılacak, 3. Bölüm'de evrim ve bağ denklemleri aracılığıyla $f(R, T)$ gravitede dönen Bianchi tip-II ve dönen Bianchi tip-V modellerinin alan denklemleri elde edilecek ve bunların çözümleri araştırılacak, 4. Bölüm'de ise yapılanlar özetlenecek ve bulunanlar tartışılacaktır.

Bu çalışamada, $c=8 \pi G=1$ doğal birim sistemi kullanılacaktır. Latin indisleri $a, b, c, \ldots$ $0,1,2,3$; Greek indisleri $\mu, v, \rho, \ldots$ ise $1,2,3$ değerlerini almakta ve (') zamana göre türevi göstermektedir.

\section{F(R,T) GRAVITENIN ALAN DENKLEMLERI}

Çok iyi bilindiği üzere Einstein Alan Denklemlerinin (EAD) geometrik kısmı, $R$ Ricci skaler eğriliğine göre lineer olan,

$$
S_{E H}=\int d^{4} x \sqrt{-g} R
$$

biçimindeki Einstein-Hilbert (EH) aksiyonundan, metriğe göre bir varyasyonla elde edilebilmektedir. Aksiyonun, $R$ Ricci skaler eğriliği yerine $R$ nin ve enerji-momentum tansörünün izi $T$ nin $f(R, T)$ gibi keyfi bir fonksiyonu alınarak

$$
S=\frac{1}{2} \int d^{4} x \sqrt{-g}\left[f(R, T)+2 L_{m}\right]
$$

biçiminde değiştirilmesiyle elde edilen teoriye $f(R, T)$ gravitasyon teorisi denir [25]. (2) aksiyonunda, $L_{m}$ maddenin Lagrange yoğunluğu olup bilinen maddenin enerji-momentum tansörü 


$$
T_{a b}^{m}=\frac{2}{\sqrt{-g}} \frac{\delta\left(\sqrt{-g} L_{m}\right)}{\delta g^{a b}}=\mu^{m} u_{a} u_{b}+p^{m} h_{a b}+q_{a}^{m} u_{b}+q_{b}^{m} u_{a}+\pi_{a b}^{m}
$$

ile verilmektedir. Burada $\mu^{m}, p^{m}, q_{a}^{m}$ ve $\pi_{a b}^{m}$, sirasıyla, bilinen maddenin enerji yoğunluğu, basıncı, 1sı akısı ve eşyönsüz basıncı; $u_{a}$ akışkanla birlikte hareket eden gözlemcinin 4-lü hız vektörü $\left(u_{a} u^{a}=-1\right)$ ve $h_{a b}$ de, metrik tansör $g_{a b}$ ve 4-lü hız vektörü $u_{a}$ aracılığıyla, $h_{a b} \equiv g_{a b}+u_{a} u_{b} \quad$ biçiminde tanımlanan, gözlemcinin 3-boyutlu durgunluk uzayına dik izdüşürme operatörüdür.

(2) aksiyonun $g_{a b}$ metriğine göre varyasyonu, $f(R, T)$ gravitasyon teorisinin

$$
f_{R}(R, T) R_{a b}-\frac{1}{2} f(R, T) g_{a b}+\left(g_{a b} \square-\nabla_{a} \nabla_{b}\right) f_{R}(R, T)=T_{a b}^{m}-f_{T}(R, T)\left(T_{a b}^{m}+\Theta_{a b}\right)
$$

biçimindeki alan denklemlerine yol açar. Burada $f_{R}(R, T)$ ve $f_{T}(R, T), f_{R}(R, T)=\frac{\partial f(R, T)}{\partial R}$ ve $f_{T}(R, T)=\frac{\partial f(R, T)}{\partial T}$ türevlerini; $\nabla_{a}$ 4-boyutta kovaryant türev operatörü olmak üzere, $\square \equiv g^{a b} \nabla_{a} \nabla_{b}=\nabla_{a} \nabla^{a} \quad$ ise $\quad$ 4-boyutlu D'Alambert operatörünü göstermektedir ve $\Theta_{a b}=-2 T_{a b}^{m}+g_{a b} L_{m}-2 g^{m n} \frac{\partial^{2} L_{m}}{\partial g^{m n} \partial g^{a b}}$ dir. Eğer evreni dolduran madde-enerji içeriğinin bir mükemmel akışkan olduğu varsayılırsa, yani, $q_{a}^{m}=0=\pi_{a b}^{m}$ olursa, maddenin enerjimomentum tansörü (3)

$$
T_{a b}^{m}=\left(\mu^{m}+p^{m}\right) u_{a} u_{b}+p^{m} g_{a b}
$$

haline indirgenir ve maddenin Lagrange yoğunluğu $L_{m}=p^{m}$ olarak alınabilir. $\mathrm{Bu}$ takdirde, $\Theta_{a b}$ nin açık ifadesi

$$
\Theta_{a b}=-2 T_{a b}^{m}+g_{a b} p^{m}
$$

olur. $\mathrm{Bu},(4)$ ile verilen alan denklemlerinde kullanılırsa, $f(R, T)$ gravitenin alan denklemleri için

$$
f_{R}(R, T) R_{a b}-\frac{1}{2} f(R, T) g_{a b}+\left(g_{a b} \square-\nabla_{a} \nabla_{b}\right) f_{R}(R, T)=T_{a b}^{m}+f_{T}(R, T)\left(T_{a b}^{m}-g_{a b} p^{m}\right)
$$

elde edilir. Bu denklem, eşitliğin sol tarafında $G_{a b} \equiv R_{a b}-\frac{1}{2} g_{a b} R$ Einstein tansörünü ortaya çıkarmak için, 


$$
\begin{aligned}
G_{a b} \equiv R_{a b}-\frac{1}{2} & R g_{a b}=\frac{T_{a b}^{m}}{f_{R}(R, T)}+\frac{f_{T}(R, T)}{f_{R}(R, T)}\left(T_{a b}^{m}-g_{a b} p^{m}\right) \\
& +\frac{1}{f_{R}(R, T)}\left\{\frac{1}{2}\left[f(R, T)-R f_{R}(R, T)\right] g_{a b}-\left(g_{a b} \square-\nabla_{a} \nabla_{b}\right) f_{R}(R, T)\right\}
\end{aligned}
$$

biçiminde yazılabilir. Eğer, literatürde sıklıkla kullanıldığı gibi, (8) denkleminin sağ tarafına efektif enerji-momentum tansörü yaklaşımını uygulamak için,

$$
\begin{aligned}
T_{a b}^{t}=\frac{T_{a b}^{m}}{f_{R}(R, T)}+\frac{f_{T}(R, T)}{f_{R}(R, T)}\left(T_{a b}^{m}-g_{a b} p^{m}\right) \\
\quad+\frac{1}{f_{R}(R, T)}\left\{\frac{1}{2}\left[f(R, T)-R f_{R}(R, T)\right] g_{a b}-\left(g_{a b} \square-\nabla_{a} \nabla_{b}\right) f_{R}(R, T)\right\}
\end{aligned}
$$

biçiminde bir $T_{a b}^{t}$ toplam efektif enerji momentum tansörü tanımlanırsa, (8) denklemi, $G_{a b}=T_{a b}^{t}$ standart EAD formunda yazılmış olur. Hemen görüleceği üzere, (8) denklemi, $f(R, T) \equiv f(R)$ alındığında $f(R)$-gravitenin alan denklemlerine indirgenmektedir [23].

Literatürde, $f(R, T)$ fonksiyonunun çeşitli özel halleri göz önüne alınagelmektedir [25]. Biz bu çalışmada, $f(R, T)$ fonksiyonunun

$$
f(R, T)=R+f(T)
$$

fonksiyonel formunu göz önüne alacağız. $f(R, T)=R+f(T)$ için (8) ile verilen alan denklemleri

$$
G_{a b}=\left[1+f_{T}(T)\right] T_{a b}^{m}+\frac{1}{2}\left[f(T)-2 p^{m} f_{T}(T)\right] g_{a b}
$$

halini alır. Bu durumda (11) efektif toplam enerji-momentum tansörünün

$$
T_{a b}^{t} \equiv\left[1+f_{T}(T)\right] T_{a b}^{m}+\frac{1}{2}\left[f(T)-2 p^{m} f_{T}(T)\right] g_{a b}
$$

olacağı hemen görülmektedir.

$T_{a b}^{t}$ efektif toplam enerji-momentum tansörü de, (3) e benzer şekilde

$$
T_{a b}^{t}=\mu^{t} u_{a} u_{b}+p^{t} h_{a b}+q_{a}^{t} u_{b}+q_{b}^{t} u_{a}+\pi_{a b}^{t}
$$

biçiminde ayrıştırılabilir. Burada, $\mu^{t}, p^{t}, q_{a}^{t}$ ve $\pi_{a b}^{t}$ efektif toplam dinamik büyüklüklerdir ve sırasıyla, efektif toplam enerji yoğunluğu, efektif toplam basınç, efektif toplam ısı akısı ve 
efektif toplam eşyönsüz basınç olarak adlandırılırlar. Öte yandan, dinamik büyüklükler (13) formunda verilmiş herhangi bir enerji-momentum tansöründen

$$
\mu=u^{a} u^{b} T_{a b}, \quad p=\frac{1}{3} h^{a b} T_{a b}, \quad q_{a}=-h_{a}^{b} u^{c} T_{b c}, \quad \pi_{a b}=h_{\langle a}^{c} h_{b\rangle}^{d} T_{c d}
$$

bağıntıları aracılığıyla bulunabilirler.

Şimdi (12), (13) ve (14) denklemleri kullanılırsa, $f(R, T)=R+f(T)$ gravitenin efektif toplam dinamik büyüklüklerinin ifadeleri için

$$
\begin{aligned}
& \mu^{t}=\mu^{m}-\frac{1}{2} f(T)+f_{T}(T)\left(\mu^{m}+p^{m}\right) \\
& p^{t}=p^{m}+\frac{1}{2} f(T) \\
& q_{a}^{t}=0 \\
& \pi_{a b}^{t}=0
\end{aligned}
$$

elde edilir.

\section{DÖNEN BIANCHİ TIP-II VE DÖNEN BIANCHI TIPP-V MODELLERİ}

$\omega^{a}$ bir-formlar tabanında aşağıdaki metrik ile verilen, uzaysal olarak homojen dönen Bianchi-tip II ve dönen Bianchi-tip V modellerini göz önüne alıyoruz.

$$
d s^{2}=-\left(\omega^{0}-a(t) v_{\alpha} \omega^{\alpha}\right)^{2}+a^{2}(t) k_{\alpha}^{2}\left(\omega^{\alpha}\right)^{2}
$$

Burada $a(t)$ zamana bağlı ölçek çarpanı, $k_{\alpha}>0$ ile $v_{\alpha}$ sabit parametrelerdir ve dönmenin sıfırdan farklı olabilmesi için en az bir $v_{\alpha}$ parametresinin sıfırdan farklı olması gerekmektedir. Bir ortonormal tabanda çalışmak çok daha kolay olduğu için, şimdi, $\omega^{a}$ tabanından, $\eta_{a b}=\operatorname{diag}(-1,1,1,1)$ olmak üzere, uzay-zaman elemanının $d s^{2}=\eta_{a b} \sigma^{a} \sigma^{b}$ şeklini alacağı bir ortonormal eş hareketli $\left(e_{0}=\mathbf{u}\right)$ tetrad çatısına geçiyoruz. $\sigma^{a}$ ortonormal birformları, (16) ifadesinden şu aşağıdaki aşikâr seçimle gerçekleştirilebilir:

$$
\sigma^{0}=\omega^{0}-a(t) v_{\alpha} \omega^{\alpha} \quad, \quad \sigma^{\alpha}=a(t) k_{\alpha} \omega^{\alpha}
$$

Böyle bir eş hareketli ortonormal tetrad çatısı için $e_{a}$ taban vektörleri ise $\sigma^{a}\left(e_{b}\right)=\delta_{b}^{a}$ dualite bağıntısı aracılığıyla tanımlanır ve $e_{a}$ taban vektörlerinin komütatörleri, $e_{0}$ a göre ölçülen akışkanın kinematik büyüklükleri cinsinden aşağıdaki gibi verilmiştir: 


$$
\begin{aligned}
& {\left[e_{0}, e_{\beta}\right]=\dot{u}_{\beta} e_{0}+\left[\varepsilon_{\beta \delta}^{\gamma}\left(\Omega^{\delta}+\omega^{\delta}\right)-\sigma_{\beta}^{\gamma}-\frac{1}{3} \delta_{\beta}^{\gamma} \theta\right] e_{\gamma}} \\
& {\left[e_{\alpha}, e_{\beta}\right]=2 \varepsilon_{\alpha \beta \gamma} \omega^{\gamma} e_{0}+\left(\varepsilon_{\alpha \beta \delta} n^{\gamma \delta}+a_{\alpha} \delta_{\beta}^{\gamma}-a_{\beta} \delta_{\alpha}^{\gamma}\right) e_{\gamma}}
\end{aligned}
$$

Burada, akışkanın kinematik büyüklükleri, $\dot{u}_{a}, \theta, \quad \sigma_{a b}\left(\sigma^{2}=(1 / 2) \sigma_{a b} \sigma^{a b}\right)$ ve $\omega^{a}$ $\left(\omega^{2}=\omega_{a} \omega^{a}\right)$ dir ve bunlar sırasıyla; ivme, genişleme, makaslama ve dönme (girdap) olarak adlandırılırlar. $n_{\alpha \beta}$ ile $a_{\alpha}$ komütasyon büyüklükleri ve $\Omega_{\alpha}$ ise $\left\{e_{\alpha}\right\}$ uzaysal tabanının yerel açısal hızıdır. Bu konuda daha ayrıntılı bilgi için [26,27] çalışmasına bakılabilir.

\subsection{Dönen Bianchi tip-II modeli}

Bianchi tip-II modeli $\omega^{a}$ bir-formlar tabanında,

$$
\omega^{a}=(d t, d x-z d y, d y, d z)
$$

koordinat temsili ile tanımlanmıştır [28]. (17) koordinat temsili için, ortonormal bir-formlar (16) metriği ve (17) seçimi uyarınca

$$
\begin{aligned}
& \sigma^{0}=d t-v_{1} a(t) d x+\left(v_{1} z-v_{2}\right) a(t) d y-v_{3} a(t) d z \\
& \sigma^{1}=k_{1} a(t) d x-k_{1} a(t) z d y \\
& \sigma^{2}=k_{2} a(t) d y \\
& \sigma^{3}=k_{3} a(t) d z,
\end{aligned}
$$

biçiminde seçilebilir. $\mathrm{Bu}$ ortonormal çatının taban vektörleri de $\sigma^{a}\left(e_{b}\right)=\delta_{b}^{a}$ dualite bağıntısını sağlayacak şekilde, aşağıdaki gibi elde edilir:

$$
\begin{aligned}
& e_{0}=\partial_{t} \\
& e_{1}=\frac{v_{1}}{k_{1}} \partial_{t}+\frac{1}{k_{1} a(t)} \partial_{x} \\
& e_{2}=\frac{v_{2}}{k_{2}} \partial_{t}+\frac{z}{k_{2} a(t)} \partial_{x}+\frac{1}{k_{2} a(t)} \partial_{y} \\
& e_{3}=\frac{v_{3}}{k_{3}} \partial_{t}+\frac{1}{k_{3} a(t)} \partial_{z}
\end{aligned}
$$

(21) ile verilen $e_{a}$ taban vektörleri (18.a) ve (18.b) komütasyon bağıntılarında kullanılarak akışkanın kinematik büyüklükleri 


$$
\begin{aligned}
& \sigma_{\alpha \beta}=0, \quad \theta=3 \frac{\dot{a}}{a}, \quad \dot{u}_{\alpha}=\frac{v_{\alpha}}{k_{\alpha}} \frac{\dot{a}}{a}, \quad \omega_{\alpha}=\left(\frac{v_{1}}{2 k_{2} k_{3}} \frac{1}{a}, 0,0,\right)=-\Omega_{\alpha} \\
& a_{\alpha}=-\frac{v_{\alpha}}{k_{\alpha}} \frac{\dot{a}}{a}, \quad n_{\alpha \beta}=\operatorname{diag}\left(-\frac{k_{1}}{k_{2} k_{3}} \frac{1}{a}, 0,0\right)
\end{aligned}
$$

olarak bulunur.

$\mathrm{Bu}$ çalışmada (21) taban vektörlerini ve (22) kinematik büyüklüklerini kullanarak akışkanın efektif toplam dinamik büyüklüklerini hesaplamak için, alan denklemlerinin bileşenlerini kullanmak yerine, bunlara denk olan tetrad evrim ve bağ denklemlerini kullanacağız. $f(R, T)$ gravitenin tetrad evrim ve bağ denklemleri rölativist kozmolojininkilerle [26] aynı formda olacaktır. Ancak, evrim ve bă̆ denklemlerini $f(R, T)$ graviteye genelleştirmek için Rölativist Kozmolojinin evrim ve bağ denklemlerinde $\mu, p$, $q_{\alpha}$ ve $\pi_{\alpha \beta}$ yerine $\mu^{t}, p^{t}, q_{\alpha}^{t}$ ve $\pi_{\alpha \beta}^{t}$ ifadeleri yazılacak ve $\nabla^{a} G_{a b}=0$ Bianchi özdeşliğinin bir sonucu olan $\nabla^{a} T_{a b}^{t}=0$ dan kaynaklanan efektif toplam madde korunum denklemlerine ek olarak, bilinen maddenin korunumunu da incelemek gerekecektir. Söz konusu korunum denklemi ile daha sonra ilgilenmek üzere, bu söylenenler 1şı̆̆ında [24] çalışmasında listelenen $f(R, T)$ graviteye uyarlanmış tetrad evrim ve bağ denklemlerinden Bianchi-tip II modeli için efektif toplam dinamik büyüklükleri aşağıdaki gibi buluyoruz:

$$
\begin{aligned}
& \mu^{t}=-\left(\frac{v_{1}^{2}}{k_{1}^{2}}+\frac{v_{2}^{2}}{k_{2}^{2}}+\frac{v_{3}^{2}}{k_{3}^{2}}\right)\left(2 \frac{\ddot{a}}{a}+\frac{\dot{a}^{2}}{a^{2}}\right)+3 \frac{\dot{a}^{2}}{a^{2}}-\frac{k_{1}^{2}-3 v_{1}^{2}}{4 k_{2}^{2} k_{3}^{2}} \frac{1}{a^{2}} \\
& p^{t}=\frac{1}{3}\left(\frac{v_{1}^{2}}{k_{1}^{2}}+\frac{v_{2}^{2}}{k_{2}^{2}}+\frac{v_{3}^{2}}{k_{3}^{2}}\right)\left(4 \frac{\ddot{a}}{a}+5 \frac{\dot{a}^{2}}{a^{2}}\right)-\left(2 \frac{\ddot{a}}{a}+\frac{\dot{a}^{2}}{a^{2}}\right)+\frac{k_{1}^{2}+v_{1}^{2}}{12 k_{2}^{2} k_{3}^{2}} \frac{1}{a^{2}} \\
& q_{1}^{t}=2 \frac{v_{1}}{k_{1}}\left(\frac{\ddot{a}}{a}-\frac{\dot{a}^{2}}{a^{2}}\right)-\frac{k_{1} v_{1}}{2 k_{2}^{2} k_{3}^{2}} \frac{1}{a^{2}} \\
& q_{2}^{t}=2 \frac{v_{2}}{k_{2}}\left(\frac{\ddot{a}}{a}-\frac{\dot{a}^{2}}{a^{2}}\right)-\frac{v_{1} v_{3}}{k_{2} k_{3}^{2}} \frac{\dot{a}}{a^{2}} \\
& q_{3}^{t}=2 \frac{v_{3}}{k_{3}}\left(\frac{\ddot{a}}{a}-\frac{\dot{a}^{2}}{a^{2}}\right)+\frac{v_{1} v_{2}}{k_{2}^{2} k_{3}} \frac{\dot{a}}{a^{2}} \\
& \pi_{11}^{t}=\frac{2}{3}\left(-2 \frac{v_{1}^{2}}{k_{1}^{2}}+\frac{v_{2}^{2}}{k_{2}^{2}}+\frac{v_{3}^{2}}{k_{3}^{2}}\right)\left(\frac{\ddot{a}}{a}-\frac{\dot{a}^{2}}{a^{2}}\right)+\frac{2 k_{1}^{2}-v_{1}^{2}}{3 k_{2}^{2} k_{3}^{2}} \frac{1}{a^{2}} \\
& \pi_{22}^{t}=\frac{2}{3}\left(\frac{v_{1}^{2}}{k_{1}^{2}}-2 \frac{v_{2}^{2}}{k_{2}^{2}}+\frac{v_{3}^{2}}{k_{3}^{2}}\right)\left(\frac{\ddot{a}}{a}-\frac{\dot{a}^{2}}{a^{2}}\right)-\frac{2 k_{1}^{2}-v_{1}^{2}}{6 k_{2}^{2} k_{3}^{2}} \frac{1}{a^{2}}
\end{aligned}
$$




$$
\begin{aligned}
& \pi_{33}^{t}=\frac{2}{3}\left(\frac{v_{1}^{2}}{k_{1}^{2}}+\frac{v_{2}^{2}}{k_{2}^{2}}-2 \frac{v_{3}^{2}}{k_{3}^{2}}\right)\left(\frac{\ddot{a}}{a}-\frac{\dot{a}^{2}}{a^{2}}\right)-\frac{2 k_{1}^{2}-v_{1}^{2}}{6 k_{2}^{2} k_{3}^{2}} \frac{1}{a^{2}} \\
& \pi_{12}^{t}=-2 \frac{v_{1} v_{2}}{k_{1} k_{2}}\left(\frac{\ddot{a}}{a}-\frac{\dot{a}^{2}}{a^{2}}\right)+\frac{k_{1} v_{3}}{k_{2} k_{3}^{2}} \frac{\dot{a}}{a^{2}} \\
& \pi_{23}^{t}=-2 \frac{v_{2} v_{3}}{k_{2} k_{3}}\left(\frac{\ddot{a}}{a}-\frac{\dot{a}^{2}}{a^{2}}\right) \\
& \pi_{31}^{t}=-2 \frac{v_{3} v_{1}}{k_{3} k_{1}}\left(\frac{\ddot{a}}{a}-\frac{\dot{a}^{2}}{a^{2}}\right)+\frac{k_{1} v_{2}}{k_{2}^{2} k_{3}} \frac{\dot{a}}{a^{2}}
\end{aligned}
$$

Şimdi, (23)-(26) efektif toplam dinamik büyüklükleri ile (15) ifadeleri birbirlerine eşitlenirse şu aşağıdaki denklem sistemine ulaşı1ır:

$$
\begin{aligned}
& -\left(\frac{v_{1}^{2}}{k_{1}^{2}}+\frac{v_{2}^{2}}{k_{2}^{2}}+\frac{v_{3}^{2}}{k_{3}^{2}}\right)\left(2 \frac{\ddot{a}}{a}+\frac{\dot{a}^{2}}{a^{2}}\right)+3 \frac{\dot{a}^{2}}{a^{2}}-\frac{k_{1}^{2}-3 v_{1}^{2}}{4 k_{2}^{2} k_{3}^{2}} \frac{1}{a^{2}}=\mu^{m}-\frac{1}{2} f(T)+f_{T}(T)\left(\mu^{m}+p^{m}\right) \\
& \frac{1}{3}\left(\frac{v_{1}^{2}}{k_{1}{ }^{2}}+\frac{v_{2}{ }^{2}}{k_{2}{ }^{2}}+\frac{v_{3}{ }^{2}}{k_{3}{ }^{2}}\right)\left(4 \frac{\ddot{a}}{a}+5 \frac{\dot{a}^{2}}{a^{2}}\right)-\left(2 \frac{\ddot{a}}{a}+\frac{\dot{a}^{2}}{a^{2}}\right)+\frac{k_{1}^{2}+v_{1}{ }^{2}}{12 k_{2}{ }^{2} k_{3}{ }^{2}} \frac{1}{a^{2}}=p^{m}+\frac{1}{2} f(T) \\
& 2 \frac{v_{1}}{k_{1}}\left(\frac{\ddot{a}}{a}-\frac{\dot{a}^{2}}{a^{2}}\right)-\frac{k_{1} v_{1}}{2 k_{2}^{2} k_{3}^{2}} \frac{1}{a^{2}}=0 \\
& 2 \frac{v_{2}}{k_{2}}\left(\frac{\ddot{a}}{a}-\frac{\dot{a}^{2}}{a^{2}}\right)-\frac{v_{1} v_{3}}{k_{2} k_{3}^{2}} \frac{\dot{a}}{a^{2}}=0 \\
& 2 \frac{v_{3}}{k_{3}}\left(\frac{\ddot{a}}{a}-\frac{\dot{a}^{2}}{a^{2}}\right)+\frac{v_{1} v_{2}}{k_{2}^{2} k_{3}} \frac{\dot{a}}{a^{2}}=0 \\
& \frac{2}{3}\left(-2 \frac{v_{1}^{2}}{k_{1}^{2}}+\frac{v_{2}^{2}}{k_{2}^{2}}+\frac{v_{3}^{2}}{k_{3}^{2}}\right)\left(\frac{\ddot{a}}{a}-\frac{\dot{a}^{2}}{a^{2}}\right)+\frac{2 k_{1}^{2}-v_{1}^{2}}{3 k_{2}^{2} k_{3}^{2}} \frac{1}{a^{2}}=0 \\
& \frac{2}{3}\left(\frac{v_{1}^{2}}{k_{1}^{2}}-2 \frac{v_{2}^{2}}{k_{2}^{2}}+\frac{v_{3}^{2}}{k_{3}^{2}}\right)\left(\frac{\ddot{a}}{a}-\frac{\dot{a}^{2}}{a^{2}}\right)-\frac{2 k_{1}^{2}-v_{1}^{2}}{6 k_{2}^{2} k_{3}^{2}} \frac{1}{a^{2}}=0 \\
& \frac{2}{3}\left(\frac{v_{1}^{2}}{k_{1}^{2}}+\frac{v_{2}^{2}}{k_{2}^{2}}-2 \frac{v_{3}^{2}}{k_{3}^{2}}\right)\left(\frac{\ddot{a}}{a}-\frac{\dot{a}^{2}}{a^{2}}\right)-\frac{2 k_{1}^{2}-v_{1}^{2}}{6 k_{2}^{2} k_{3}^{2}} \frac{1}{a^{2}}=0
\end{aligned}
$$




$$
\begin{aligned}
& -2 \frac{v_{1} v_{2}}{k_{1} k_{2}}\left(\frac{\ddot{a}}{a}-\frac{\dot{a}^{2}}{a^{2}}\right)+\frac{k_{1} v_{3}}{k_{2} k_{3}^{2}} \frac{\dot{a}}{a^{2}}=0 \\
& -2 \frac{v_{2} v_{3}}{k_{2} k_{3}}\left(\frac{\ddot{a}}{a}-\frac{\dot{a}^{2}}{a^{2}}\right)=0 \\
& -2 \frac{v_{3} v_{1}}{k_{3} k_{1}}\left(\frac{\ddot{a}}{a}-\frac{\dot{a}^{2}}{a^{2}}\right)+\frac{k_{1} v_{2}}{k_{2}^{2} k_{3}} \frac{\dot{a}}{a^{2}}=0
\end{aligned}
$$

$\mathrm{Bu}$ denklemlerin birbirleriyle tutarlılığını incelemek için aşağıdaki üç durumu göz önüne aliyoruz:

1. $v_{1} \neq 0, v_{2} \neq 0, v_{3} \neq 0: \mathrm{Bu}$ durumda gerekli sadeleştirmelerden sonra (30.e) denklemi $\left(\frac{\ddot{a}}{a}-\frac{\dot{a}^{2}}{a^{2}}\right)=0$ verir. $\mathrm{Bu}(29 . \mathrm{a})$ ya yerleştirildiğinde $k_{1} /\left(2 k_{2}{ }^{2} k_{3}{ }^{2} a^{2}\right)=0$ a yol açar ve buradan da $k_{1}=0$ bulunur ki, bu $k_{1} \neq 0$ kabulüyle çelişir.

2. $v_{1} \neq 0, v_{2} \neq 0, v_{3}=0$ : Yukarıdaki duruma benzer şekilde, bu durumda da (30.d) denklemi $\left(\frac{\ddot{a}}{a}-\frac{\dot{a}^{2}}{a^{2}}\right)=0$ verir ve bu $k_{1} \neq 0$ olduğundan (29.a) ile çelişir.

3. $v_{1} \neq 0, v_{2}=0, v_{3}=0: \mathrm{Bu}$ durumda, (29.b), (29.c) ve (30.d)-(30.f) denklemleri özdeş olarak sağlanırken, (29.a),

$$
\left(\frac{\ddot{a}}{a}-\frac{\dot{a}^{2}}{a^{2}}\right)=\frac{k_{1}^{2}}{4 k_{2}^{2} k_{3}^{2}} \frac{1}{a^{2}}
$$

denklemine ve (30.a)-(30.c) denklemleri de tek bir

$$
\frac{v_{1}^{2}}{k_{1}^{2}}\left(\frac{\ddot{a}}{a}-\frac{\dot{a}^{2}}{a^{2}}\right)=\frac{2 k_{1}^{2}-v_{1}^{2}}{4 k_{2}^{2} k_{3}^{2}} \frac{1}{a^{2}}
$$

denklemine indirgenir. (31) denklemi, (32) ye yerleştirilirse tutarlılık koşulu olarak $v_{1}^{2}=k_{1}^{2}$ elde edilir. Şimdi, $\left(\mu^{m}+p^{m}\right) \neq 0$ varsayımının sağlanıp sağlanmadığını kontrol edelim. Bunun için $v_{1}^{2}=k_{1}^{2}$ koşulu altında, (23) ve (24) denklemlerinin aşağıdaki kombinasyonunu göz önüne alalım:

$$
-\frac{8}{3}\left(\frac{\ddot{a}}{a}-\frac{\dot{a}^{2}}{a^{2}}\right)+\frac{2 k_{1}^{2}}{3 k_{2}^{2} k_{3}^{2}} \frac{1}{a^{2}}=\frac{1}{2}\left[1+f_{T}(T)\right]\left(\mu^{m}+p^{m}\right)
$$

Bu son denklem, (31) kullanılırsa 


$$
\left[1+f_{T}(T)\right]\left(\mu^{m}+p^{m}\right)=0
$$

halini alır. Buradan kolayca, $\left(\mu^{m}+p^{m}\right) \neq 0$ kabulünden dolayı, $1+f_{T}=0$ olması gerektiği anlaşılmaktadır ve bunun integrasyonu $c$ integrasyon sabiti olmak üzere $f(T)$ fonksiyonunun

$$
f(T)=-T+c
$$

fonksiyonel formunu verir ve bu durumda $f(R, T)=R+f(T)$ fonksiyonu

$$
f(R, T)=R-T+c
$$

biçiminde tamamen belirlenmiş olur. Öte yandan, (31) diferansiyel denklemi de, $c_{1}$ ve $c_{2}$ integrasyon sabitleri olmak üzere,

$$
a(t)=\frac{k_{1}}{2 k_{2} k_{3} c_{1}} \cosh \left(c_{1} t+c_{2}\right)
$$

biçiminde integre edilebilir. Böylece, $f(R, T)=R+f(T)$ gravitede aynı anda hem dönen ve hem de genişleyen Bianchi-tip II evren modeli için, zamana bağlı bir ölçek çarpanı ve $f(R, T)$ fonksiyonunun fonksiyonel formunu bulmuş oluyoruz.

Şimdi, bu evrenin madde-enerji içeriği hakkında bilgi sahibi olabilmek için, yukarıda söz ettiğimiz korunum denklemini inceleyelim. Mükemmel akışkan için enerji momentum tansörünün ifadesi (5) ten kolayca elde edilebilecek olan $T=-\mu^{m}+3 p^{m}$ ile (35.a) veya (35.b) fonksiyonu, (12) ile verilen efektif toplam enerji-momentum tansöründe kullanılıp, bunun da kovaryant türevi alınırsa, $\nabla^{a} T_{a b}^{t}=0$ olduğundan

$$
\nabla_{a}\left(\mu^{m}-p^{m}\right)=0
$$

olması gerektiği bulunur ve bunun da integrasyonu, $c_{3}$ integrasyon sabiti olmak üzere,

$$
p^{m}=\mu^{m}+c_{3}
$$

biçimindeki barotropik hal denklemini verir. Buradan $c_{3}$ sabitinin değerlerine göre $\mu^{m}>0$ doğal koşuluna uyan herhangi bir madde için fiziksel olan pozitif basınca karşılık gelen durumlar olduğu hemen görülmektedir. (38) hal denklemi özel olarak $c_{3}=0$ hali için, $w=1$ olmak üzere, $p^{m}=w \mu^{m}(-1 \leq w \leq 1)$ lineer barotropik hal denklemine indirgenmektedir ki bu, evreni dolduran madde içeriğinin bir katı akışkan olduğu anlamına gelmektedir.

\subsection{Dönen Bianchi tip-V modeli}

Yukarıdaki alt paragrafta, Bianchi-tip II için yapılanları şimdi koordinat temsili 


$$
\omega^{a}=\left(d t, d x, e^{x} d y, e^{x} d z\right)
$$

ile verilen [28], Bianchi tip-V modeli için tekrarlayacağız. Bu model için $\sigma^{a}$ bir-formları ve $e_{a}$ taban vektörleri

$$
\begin{array}{ll}
\sigma^{0}=d t-v_{1} a(t) d x-v_{2} a(t) e^{x} d y-v_{3} a(t) e^{x} d z, & e_{0}=\partial_{t} \\
\sigma^{1}=k_{1} a(t) d x, & e_{1}=\frac{v_{1}}{k_{1}} \partial_{t}+\frac{1}{k_{1} a(t)} \partial_{x} \\
\sigma^{2}=k_{2} a(t) e^{x} d y, & e_{2}=\frac{v_{2}}{k_{2}} \partial_{t}+\frac{e^{-x}}{k_{2} a(t)} \partial_{y} \\
\sigma^{3}=k_{3} a(t) e^{x} d z, & e_{3}=\frac{v_{3}}{k_{3}} \partial_{t}+\frac{e^{-x}}{k_{3} a(t)} \partial_{z}
\end{array}
$$

biçiminde olacaktır. Bu taban vektörleri (18) komütasyon bağıntılarında kullanılarak akışkanın kinematik büyüklükleri için

$$
\begin{aligned}
& \sigma_{\alpha \beta}=0, \quad \theta=3 \frac{\dot{a}}{a}, \quad \dot{u}_{\alpha}=\frac{v_{\alpha}}{k_{\alpha}} \frac{\dot{a}}{a}, \quad \omega_{\alpha}=\left(0,-\frac{v_{3}}{2 k_{3} k_{1}} \frac{1}{a}, \frac{v_{2}+v_{3}}{2 k_{1} k_{2}} \frac{1}{a}\right)=-\Omega_{\alpha}, \\
& a_{\alpha}=-\frac{v_{\alpha}}{k_{\alpha}} \frac{\dot{a}}{a}-\frac{1}{k_{\alpha} a} \delta_{1 \alpha}, \quad n_{\alpha \beta}=\operatorname{diag}\left(0,0,-\frac{k_{3}}{k_{1} k_{2}} \frac{1}{a}\right)
\end{aligned}
$$

bulunur. Taban vektörleri ve kinematik büyüklüklerin tetrad evrim ve bağ denklemlerinde kullanılmasıyla elde edilebilecek olan efektif toplam dinamik büyüklükler ve (15) bağıntıları aracılığıyla dönen Bianchi-tip V modeli için şu aşă̆ıdaki denklem sistemi elde edilir:

$$
\begin{aligned}
& -\frac{2}{3}\left(\frac{v_{1}^{2}}{k_{1}^{2}}+\frac{v_{2}^{2}}{k_{2}^{2}}+\frac{v_{3}^{2}}{k_{3}^{2}}\right)\left(\frac{\ddot{a}}{a}-\frac{\dot{a}^{2}}{a^{2}}\right)-\frac{4}{3} \frac{v_{1}}{k_{1}^{2}} \frac{\dot{a}}{a^{2}} \\
& +\frac{1}{k_{1}{ }^{2}}\left[-2+\frac{5}{6}\left(\frac{v_{2}{ }^{2}}{k_{2}{ }^{2}}+\frac{v_{3}{ }^{2}}{k_{3}{ }^{2}}\right)\right] \frac{1}{a^{2}}=\left[1+f_{T}(T)\right]\left(\mu^{m}+p^{m}\right) \\
& 2 \frac{v_{1}}{k_{1}}\left(\frac{\ddot{a}}{a}-\frac{\dot{a}^{2}}{a^{2}}\right)-\frac{1}{k_{1}}\left(\frac{v_{2}^{2}}{k_{2}^{2}}+\frac{v_{3}^{2}}{k_{3}^{2}}\right) \frac{\dot{a}}{a^{2}}=0 \\
& 2 \frac{v_{2}}{k_{2}}\left(\frac{\ddot{a}}{a}-\frac{\dot{a}^{2}}{a^{2}}\right)+\frac{v_{2}}{k_{2}}\left(\frac{v_{1}}{k_{1}^{2}} \frac{\dot{a}}{a^{2}}+\frac{1}{2 k_{1}^{2}}\right) \frac{1}{a^{2}}=0 \\
& 2 \frac{v_{3}}{k_{3}}\left(\frac{\ddot{a}}{a}-\frac{\dot{a}^{2}}{a^{2}}\right)+\frac{v_{3}}{k_{3}}\left(\frac{v_{1}}{k_{1}^{2}} \frac{\dot{a}}{a^{2}}+\frac{1}{2 k_{1}^{2}}\right) \frac{1}{a^{2}}=0
\end{aligned}
$$




$$
\begin{aligned}
& \left(-2 \frac{v_{1}^{2}}{k_{1}^{2}}+\frac{v_{2}^{2}}{k_{2}^{2}}+\frac{v_{3}^{2}}{k_{3}^{2}}\right)\left(\frac{\ddot{a}}{a}-\frac{\dot{a}^{2}}{a^{2}}\right)+2 \frac{v_{1}}{k_{1}^{2}} \frac{\dot{a}}{a^{2}}+\frac{1}{4 k_{1}^{2}}\left(\frac{v_{2}^{2}}{k_{2}^{2}}+\frac{v_{3}^{2}}{k_{3}^{2}}\right) \frac{1}{a^{2}}=0 \\
& \left(\frac{v_{1}^{2}}{k_{1}^{2}}-2 \frac{v_{2}^{2}}{k_{2}^{2}}+\frac{v_{3}^{2}}{k_{3}^{2}}\right)\left(\frac{\ddot{a}}{a}-\frac{\dot{a}^{2}}{a^{2}}\right)-\frac{v_{1}}{k_{1}^{2}} \frac{\dot{a}}{a^{2}}+\frac{1}{2 k_{1}^{2}}\left(\frac{1}{2} \frac{v_{2}^{2}}{k_{2}^{2}}-\frac{v_{3}^{2}}{k_{3}^{2}}\right) \frac{1}{a^{2}}=0 \\
& \left(\frac{v_{1}^{2}}{k_{1}^{2}}+\frac{v_{2}^{2}}{k_{2}^{2}}-\frac{2}{3} \frac{v_{3}^{2}}{k_{3}^{2}}\right)\left(\frac{\ddot{a}}{a}-\frac{\dot{a}^{2}}{a^{2}}\right)-\frac{v_{1}}{k_{1}^{2}} \frac{\dot{a}}{a^{2}}+\frac{1}{2 k_{1}^{2}}\left(\frac{1}{2} \frac{v_{3}^{2}}{k_{3}^{2}}-\frac{v_{2}^{2}}{k_{2}^{2}}\right) \frac{1}{a^{2}}=0 \\
& -2 \frac{v_{1} v_{2}}{k_{1} k_{2}}\left(\frac{\ddot{a}}{a}-\frac{\dot{a}^{2}}{a^{2}}\right)+\frac{v_{2}}{k_{1} k_{2}} \frac{\dot{a}}{a^{2}}=0 \\
& -2 \frac{v_{1} v_{3}}{k_{1} k_{3}}\left(\frac{\ddot{a}}{a}-\frac{\dot{a}^{2}}{a^{2}}\right)+\frac{v_{3}}{k_{1} k_{3}} \frac{\dot{a}}{a^{2}}=0 \\
& -2 \frac{v_{2} v_{3}}{k_{2} k_{3}}\left(\frac{\ddot{a}}{a}-\frac{\dot{a}^{2}}{a^{2}}\right)+\frac{1}{2} \frac{v_{2} v_{3}}{k_{1}^{2} k_{2} k_{3}} \frac{1}{a^{2}}=0
\end{aligned}
$$

$\mathrm{Bu}$ denklem sisteminin tutarlılığını da yine, $v_{\alpha}$ dönme parametrelerine göre üç durumda araştırıyoruz:

1. $v_{1} \neq 0, v_{2} \neq 0, v_{3} \neq 0$ : Bu durumda gerekli sadeleştirmelerden sonra (45), (50) ve (51) denklemleri birlikte kullanılırsa $v_{1}^{2} / 2 k_{1}^{2}=-1$ sonucunu verir ki bu çelişkili bir ifadedir.

2. $v_{1}=0, v_{2} \neq 0, v_{3} \neq 0: \mathrm{Bu}$ durum için (43) ve (49) denklemleri birlikte kullanıldığında $\dot{a}=0$ olur. Bunun da integrasyonu $a(t)=$ sabit sonucuna yol açar ki bu zamana bağlı ölçek çarpanı kabulüyle çelişir.

3. $v_{1}=0, v_{2}=0, v_{3} \neq 0:$ Bu durumda da (43) denkleminden kolayca görüleceği gibi yine $\dot{a}=0 \Rightarrow a(t)=$ sabit çelişkisine ulaşılır.

Dönen Bianchi tip-V modeli için (42)-(51) denklem sistemi, yukarıdaki her üç durumda da bir çelişkiye yol açtığından, böyle bir model $f(R, T)$ gravitenin alan denklemlerinin çözümü değildir. Başka bir deyişle Bianchi-V evreni $f(R, T)$ gravitasyon teorisi çerçevesinde aynı anda hem dönme hem de genişleme gösteremez.

\section{SONUÇLAR}

$\mathrm{Bu}$ çalışmada, değiştirilmiş gravitasyon teorilerinde makaslamasız mükemmel akışkan iddiasını yanlışlayan tek modelin uzaysal olarak homojen ve eşyönsüz Bianchi tip-IX evreni olup olmadığını araştırmak ve benzer çözümün $f(R, T)$ gravitede tüm Bianchi tip metrikler için geçerli olup olmadığını incelemek amacıyla, $f(R, T)$ gravitasyon teorisi çerçevesinde 
uzayca homojen ve eşyönsüz metrikler sınıfından olan dönen Bianchi tip-II ve dönen Bianchi tip-V modelleri göz önüne alınmıştır. Ortonormal tetrad tabanında yazılmış evrim ve bağ denklemleri kullanılarak, her iki model için elde edilen denklem sistemlerinin, metriğin parametreleri $k_{\alpha}$ ve $v_{\alpha}$ ile $a(t)$ zamana bağlı ölçek çarpanının tutarlı olup olmadığ incelenmiştir.

Bianchi tip-II modeli için $v_{1} \neq 0, v_{2}=0, v_{3}=0$ durumunda tutarlılığın sağlandığ gösterilmiş ve bu durum için (36) ile verilen bir kesin çözüm elde edilmiştir. Dahası $f(R, T)=R+f(T)$ fonksiyonun (35) ile verilen fonksiyonel şekli belirlenmiş ve böyle bir evren modelinin madde enerji içeriğini tasvir eden hal denkleminin fiziksel duruma karşılık gelen katı akışkanın hal denklemi olduğu gösterilmiştir. Başka bir deyişle, $f(R, T)$ gravitede makaslamasız katı akışkanla dolu Bianchi tip-II modelinin, aynı anda hem dönme ve hem de genişlemeye sahip olabileceği gösterilmiştir. Bu çözüm, $f(R)$ ve $f(R, T)$ gravitasyon teorilerinde, $\mu+p \neq 0$ koşuluna uyup aynı anda döndüğü ve genişlediği bilinen tek modelin Bianchi tip-IX evreni olmadığını göstermesi bakımından önem taşımaktadır.

Dönen Bianchi tip-V modeli için ise, elde edilen denklem sisteminin metriğin parametreleriyle tutarlı olmadığ çözümü olmadığı gösterilmiştir. Böyle bir çözümün var olmaması, $f(R, T)$ gravitasyon teorisinde Bianchi tip II ve IX modelleri için var olan çözümün, tüm Bianchi tip metrikler için geçerli olmadığını göstermektedir.

\section{KAYNAKLAR}

[1] Gödel K. Rotating Universes in General Relativity Theory, Proceedings of the International Congress of Mathematicians, cilt.1, 1950, s.175-181; (yeniden basim: General Relativity and Gravitation, cilt.32, 2000, s.1419).

[2] Ellis, GFR. Dynamics of Pressure-Free Matter in General Relativity, Journal of Mathematical Physics, Cilt.8, 1967, s.1171-1180.

[3] Schücking E. Homogene Scherungsfreie Weltmodelle in der Relativistischen Kosmologie, Naturwiss, cilt.19, 1957, s.507.

[4] Banerji S. Homogeneous Cosmological Models without Shear, Progress of Theoretical Physics, cilt.39, 1968, s.365-371.

[5] Treciokas R, Ellis GFR. The Shear Dynamics, Communications in Mathematical Physics, cilt.23, 1971, s.1-17.

[6] King AR, ELLIS GFR. Tilted Homogeneous Cosmological Models, Communications in Mathematical Physics, 1973, cilt.31, s.209-220.

[7] White AJ. Collins CB., A Class of Shear-free Perfect Fluids in General Relativity I, Journal of Mathematical Physics, cilt.25, 1984, s.332-337.

[8] Collins CB. Shear-free Perfect Fluids with Zero Magnetic Weyl Tensor, Journal of Mathematical Physics, cilt.25, 1984, 995-1000.

[9] Carminati J. Shear-free perfect fluids in general relativity. I. Petrov type N Weyl tensor, Journal of Mathematical Physics, cilt.28, 1987, s.1848-1853.

[10] Carminati J. Type N, Shear-free, Perfect Fluid Space-times with a Barotropic Equation of State, General Relativity and Gravitation, cilt.20, 1988, s.1239-1248.

[11] Carminati J. Shear-free Perfect Fluids in General Relativity. II. Aligned, Petrov Type III Space-times, Journal of Mathematical Physics, cilt.31, 1990, 2434-2440. 
[12] Senovilla JMM, Sopuerta CF, Szekeres P. Theorems on Shear-free Perfect Fluids with Their Newtonian Analogues, General Relativity and Gravitation, cilt.30, 1998, s.389411.

[13] Sopuerta CF. Covariant Study of a Conjecture on Shear-free Barotropic Perfect Fluids, Classical and Quantum Gravity, cilt.15, 1998, s.1043-1062.

[14] Van den Bergh N. The Shear-free Perfect Fluid Conjecture, Classical and Quantum Gravity, cilt.16, 1999, s.1-13.

[15] Van den Bergh N, Carminati J, Karimian HR. Shearfree Perfect Fluids with Solenoidal Magnetic Curvature and a Gamma-law Equation of State, Classical and Quantum Gravity, cilt.24, 2007, s.3735-3744.

[16] Carminati J. Karimian HR, Van den Bergh N, Vu KT. Shear-free Perfect Fluids with a Solenoidal Magnetic Curvature, Classical and Quantum Gravity, cilt.26, 2009, 195002.

[17] Herrera L, Di Prisco A, Ospino J. On the Stability of the Shear-free Condition, General Relativity and Gravitation, cilt.42, 2010, s.1585-1599.

[18] Herrera L, Di Prisco A, Ospino J. Shear-free Axially Symmetric Dissipative Fluids, Physical Review D, cilt.89, 2014, s.127502.

[19] Slobodeanu R. Shear-free Perfect Fluids with Linear Equation of State, Classical and Quantum Gravity, cilt.31, 2014, s.125012.

[20] Van den Bergh N, Slobodeanu R. Shear-free Perfect Fluids with a Barotropic Equation of State in General Relativity: the Present Status, arXiv, [http://arxiv.org/abs/1510.05798], 2015, Erişim Tarihi: 25.10.2015.

[21] Narlikar JV. Newtonian Universes with Shear and Rotation, Monthly Notices of the Royal Astronomical Society cilt.126, 1963, s.203.

[22] Heckmann O, Schücking E. Handbuch der Physik LIII (ed) Flügge S. Berlin: Springer, 1959, s.489.

[23] Sofuoğlu D, Mutuş H. Investigations of $f(R)$-Gravity Counterparts of the General Relativistic Shear-free Conjecture by Illustrative Examples, General Relativity and Gravitation, cilt.46, 2014, s.1-25.

[24] Sofuoğlu D. Rotating and Expanding Bianchi Type-IX Model in f(R,T) Theory of Gravity, Astrophysics and Space Science, cilt.361, 2016, s.1-7.

[25] Harko T, Lobo FSN, Nojiri S, Odintsov SD. f(R,T) Gravity, Physical Review D, cilt.84, 2011, s.024020.

[26] Ellis GFR, Van Elst H. Cosmological Models (Cargese Lectures 1998), arXiv, [http://arxiv.org/abs/gr-qc/9812046], 1998, Erişim Tarihi: 17.11.2004.

[27] Van Elst H, Uggla C. General Relativistic 1+3 Orthonormal Frame Approach, Classical and Quantum Gravity, cilt.14, 1997, s.2673-2695.

[28] MacCallum MAH. "Anisotropic and Inhomogeneous Relativistic Cosmologies" in: General Relativity An Einstein Centenary Survey, (Ed.) Hawking SW, Israel W., Cambridge: Cambridge University Press, 1979, s.179-236 (Tablo 11.2, s.194).

\section{ÖZGEÇMISS / CV}

\section{Değer SOFUOĞLU; Dr. (Ph. D.)}

Lisans derecesini İstanbul Üniversitesi Fizik Bölümü’nden 2004 y1lında, Yüksek Lisans derecesini İstanbul Üniversitesi Matematiksel Fizik Programı'ndan 2008 yılında ve Doktora derecesini de İstanbul Üniversitesi Matematiksel Fizik Doktora Programı'ndan 2014 yılında almıştır. 2006 yılından bu yana İstanbul Üniversitesi, Fen Fakültesi, Fizik Bölümü, Matematiksel Fizik Anabilim Dalı'nda Araştırma Görevlisi olarak çalışmaktadır. Çalışma konuları Matematiksel Fizik, Genel Rölativite Teorisi, Zar-evren Modelleri ve Yüksek Mertebeden 
Eğrilikli Gravitasyon Teorileridir.

She has got her bachelors' degree in Physics Department at Istanbul University in 2004, her master degree in Mathematical Physics MSc program at Istanbul University in 2008 and her PhD degree in Mathematical Physics PhD program at Istanbul University in 2014. She has been studing in Istanbul University, Science Faculty, Physics Department, Mathematical Physics Division as Research Assistant since 2006. Her research interests focus on Mathematical Physics, General Relativity Theory, Brane-world Models and Higher Order Curvature Gravity Theories. 